$\overline{\mathrm{AEET}}$

ASOCIACIÓN ESPAÑOLA

DE ECOLOGÍA TERRESTRE
Ecosistemas 21(3):15-21 [Septiembre-Diciembre 2012] Doi.: 10.7818/ECOS.2012.21-3.03

Artículo publicado en Open Access bajo los términos de Creative Commons attribution Non Comercial License.

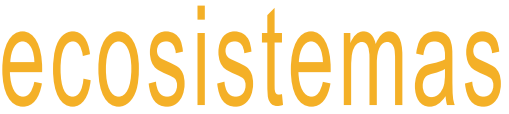

REVISTA CIENTÍFICA DE ECOLOGÍA Y MEDIO AMBIENTE

\title{
Las poblaciones ibéricas de pino albar ante el cambio climático: con la muerte en los talones
}

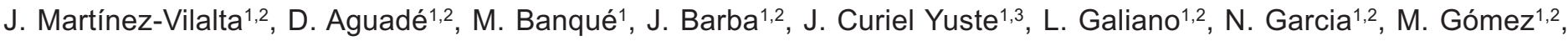 \\ A.M. Hereş ${ }^{1,2}$, B.C. López ${ }^{1,2}$, F. Lloret ${ }^{1,2}$, R. Poyatos ${ }^{1}$, J. Retana ${ }^{1,2}$, O. Sus ${ }^{1}$, J. Vayreda ${ }^{1}$, A. Vilà-Cabrera ${ }^{1,2}$
}

(1) CREAF, Cerdanyola del Vallès 08193, España.

(2) Univ. Autònoma Barcelona, Cerdanyola del Vallès 08193, España.

(3) Museo de Ciencias Naturales, CSIC, Madrid 28006, España.

*Autor de correspondencia: J. Martínez-Vilalta [Jordi.Martinez.Vilalta@uab.es]

> Recibido el 8 de octubre de 2012, aceptado el 12 de noviembre de 2012.

\begin{abstract}
Martínez-Vilalta, J., Aguadé, D., Banqué, M., Barba, J., Curiel Yuste, J., Galiano, D., Garcia, N., Gómez, M., Hereş, A.M., López, B.C., Lloret, F., Poyatos, R., Retana, J., Sus, O., Vayreda, J., Vilà-Cabrera, A. (2012). Las poblaciones ibéricas de pino albar ante el cambio climático: con la muerte en los talones. Ecosistemas 21(3):15-21. Doi.: 10.7818/ECOS.2012.21-3.03

El pino albar (Pinus sylvestris) es uno de los árboles más ampliamente distribuidos del mundo. Pese a su gran plasticidad ecológica, numerosos estudios muestran que su capacidad de resistir la sequía se está viendo superada en diversas zonas, especialmente en la cuenca mediterránea, donde se halla el límite meridional de su distribución. El presente artículo repasa una serie de trabajos recientes sobre los efectos directos e indirectos de la sequía sobre las poblaciones ibéricas de pino albar en un contexto de cambio climático. Específicamente, se tratan los siguientes aspectos: (1) ¿qué características ecofisiológicas explican la vulnerabilidad del pino albar a la sequía?; (2) ¿qué factores ambientales determinan sus patrones de crecimiento y cómo han variado en las últimas décadas?; (3) ¿qué factores ambientales explican la variabilidad espacial en las tasas demográficas de la especie (crecimiento, mortalidad, reclutamiento) a distintas escalas?; y (4) ¿cuáles son los impactos previsibles de un aumento de las condiciones de sequía y de los incendios forestales? El conjunto de los resultados registrados sugiere que una parte importante de las poblaciones ibéricas de pino albar podrían dejar de ser viables a medio plazo si se cumplen las proyecciones de cambio climático. Concluimos explorando hasta qué punto podemos utilizar la información anterior para identificar los individuos o poblaciones más vulnerables y el papel que la gestión podría tener a la hora de modular los impactos esperados.
\end{abstract}

Palabras clave: Decaimiento forestal, Dinámica forestal, Gestión forestal, Incendios, Pinus sylvestris, Sequía

Martínez-Vilalta, J., Aguadé, D., Banqué, M., Barba, J., Curiel Yuste, J., Galiano, D., Garcia, N., Gómez, M., Hereş, A.M., López, B.C., Lloret, F., Poyatos, R., Retana, J., Sus, O., Vayreda, J., Vilà-Cabrera, A. (2012). Iberian Scots pine populations under climate change: some don't like it hot. Ecosistemas 21(3):15-21. Doi.: 10.7818/ECOS.2012.21-3.03

Scots pine (Pinus sylvestris L.) is one of the most widely distributed trees on Earth. Despite its huge ecological plasticity, many studies show that its capacity to resist drought is being overcome in several regions, particularly at the southern limit of its distribution in the Mediterranean basin. This paper summarizes recent work on the direct and indirect effects of drought on Scots pine in the context of climate change. More specifically, the following aspects are addressed: (1) what are the ecophysiological characteristics that explain the vulnerability of Scots pine to drought?; (2) what environmental factors determine the growth patterns of Scots pine and how have these factors varied over the last decades?; (3) what environmental factors explain the spatial variability in the demographic rates of this species (growth, mortality, recruitment) at different scales?; and (4) what are the likely impacts of more frequent droughts and forest fires? Overall, the results currently available suggest that the mid term viability of a substantial part of the Scots pine populations in the Iberian Peninsula is at risk if climate change projections become true. We conclude exploring to what degree the previous information can be used to identify the more vulnerable individuals or populations, and how could forest management be used to modulate the expected impacts.

Keywords: Forest decline, Forest dynamics, Forest management, Wildfires, Pinus sylvestris, Drought

\section{Introducción}

Existe una preocupación creciente por las alteraciones que el cambio climático puede inducir en los ecosistemas en general y en los bosques en particular (Bonan 2008; Pan et al. 2011; Anderegg et al. 2012). Globalmente, se está detectando un número creciente de episodios de mortalidad forestal asociada a períodos de sequía o calor extremos (Allen et al. 2010). En la región mediterránea, el cambio climático está modificando las que son probablemente las dos perturbaciones 'naturales' más importantes que influencian en gran medida la distribución y el funcionamiento de la vegetación: la sequía y los incendios forestales (Lloret 2012). En este contexto, es imprescindible entender los mecanismos que determinan la vulnerabilidad de los bosques ante estos factores para poder predecir cómo nuestros bosques, y los bienes y servicios que éstos nos proporcionan, se modificarán en el futuro (cf. McDowell et al. 2011).

Una manera de evaluar el impacto que el cambio climático puede tener sobre los ecosistemas es estudiar los efectos que cam- 
bios climáticos parecidos tuvieron en el pasado o están teniendo actualmente. Para tener éxito, esta aproximación debe incluir estudios a distintas escalas espacio-temporales, abarcando desde los mecanismos ecofisiológicos subyacentes a la mortalidad (e.g., McDowell et al. 2008, McDowell 2011) hasta las respuestas demográficas de las poblaciones afectadas (Lloret et al. 2012), la variabilidad espacial en los factores ambientales y en los rasgos funcionales de las especies, así como la plasticidad de éstos y sus límites (Nicotra et al. 2010), y el impacto funcional de los cambios en la composición de las comunidades (Anderegg et al. 2012). En este artículo sintetizamos una serie de trabajos recientes sobre los impactos directos e indirectos de la sequía sobre el pino albar (Pinus sylvestris L.), centrándonos en las poblaciones ibéricas de la especie.

\section{El pino albar como modelo}

El pino albar es una de las especies arbóreas más ampliamente distribuidas del mundo, con una distribución que abarca buena parte de Eurasia. Su extensa distribución geográfica implica una gran amplitud ecológica y la capacidad de tolerar condiciones ambientales muy diversas. Así, aunque la mayor parte de poblaciones de la especie se encuentran en regiones boreales, el pino albar ocupa también grandes extensiones en zonas de clima moderadamente árido en la región mediterránea, desde la Península Ibérica a Turquía. En España es la tercera especie forestal más abundante y sus bosques cubren 1280000 ha (aproximadamente un $6 \%$ de los bosques españoles) (Mason y Alía 2000). Según el Tercer Inventario Forestal Nacional (IFN) por lo menos un $21 \%$ de los bosques de pino silvestre en España corresponden a plantaciones.

Las poblaciones ibéricas de pino albar marcan el límite meridional del área de distribución de la especie, lo cual las hace especialmente interesantes como sistemas donde estudiar los efectos de las sequías extremas, ya que las condiciones climáticas actuales de las poblaciones meridionales pueden ser representativas de las condiciones bajo las que crecerán las poblaciones más septentrionales a lo largo del presente siglo. Por otra parte, las poblaciones del límite meridional de la distribución de las especies son especialmente relevantes desde el punto de vista de la conservación de la biodiversidad, puesto que acostumbran a ser reservorios de variabilidad genética que pueden ser clave en el actual contexto de cambio climático (Hampe y Petit 2005).

La distribución del pino albar en Europa ha sufrido numerosos cambios latitudinales y altitudinales durante el Pleistoceno, ligados principalmente a los ciclos glaciales (ver Matías y Jump 2012 para una revisión reciente). Durante el último máximo glacial, hace unos 18000 años, su distribución estaba restringida, fundamentalmente, a localidades del centro y del sur de Europa, incluyendo la Península Ibérica (Willis et al. 1998; Cheddadi et al. 2006). A partir de entonces se produjo una recolonización hacia el norte a medida que las temperaturas aumentaban, hasta que el pino albar alcanzó su máxima distribución en Europa hace unos 8000 años. Posteriormente comenzó la retracción de la especie en las Penínsulas lbérica e Italiana, que culminó hace unos 3000 años y que se ha asociado a cambios en el clima pero también a impactos antrópicos (Willis et al. 1998). La actual distribución de la especie en España es en buena medida el resultado de políticas de gestión forestal que históricamente han favorecido a los pinos en detrimento de los planifolios, aunque esta tendencia parece haber cambiado en las últimas décadas (Vayreda et al., datos sin publicar).

\section{Respuesta ecofisiológica a la sequía}

No se conoce todavía el mecanismo fisiológico que conduce a la muerte de los árboles en condiciones de sequía. Se trata sin embargo de un ámbito de investigación muy activo y actualmente aceptamos que la mortalidad inducida por sequía no se puede entender sin considerar explícitamente la interacción entre los aspectos hidráulicos y la economía del carbono, así como la interacción entre éstos y los agentes bióticos (McDowell et al. 2008; Sala et al. 2010; McDowell 2011). El pino albar es una especie isohídrica; es decir, con un control estomático muy estricto de la transpiración (Irvine et al. 1998; Poyatos et al. 2007; Zweifel et al. 2007). Para este tipo de especies el modelo propuesto por McDowell et al. (2008) predice un rápido consumo de las reservas de carbohidratos, lo cual resultaría en su eventual agotamiento y en la muerte del árbol por inanición.

Los resultados de diversos estudios ecofisiológicos realizados en las montañas de Prades (Tarragona) y en el Pirineo catalán son consistentes, por lo menos en parte, con la interpretación anterior. En primer lugar demuestran que el comportamiento del pino albar es estrictamente isohídrico, en el sentido que la transpiración se reduce prácticamente a cero bajo condiciones de sequía intensa y los potenciales hídricos no parecen bajar nunca de un umbral cercano a los -2.5 MPa (Poyatos et al., datos sin publicar). Sin embargo, existe cierta variabilidad entre individuos y entre poblaciones en estos potenciales hídricos mínimos (Martínez-Vilalta et al. 2009), probablemente ligada a diferencias en la máxima sequía edáfica experimentada en cada caso.

En segundo lugar, varios trabajos muestran que la regulación del área foliar (en relación a la superficie de albura) es el principal ajuste que se produce entre poblaciones para hacer frente a la sequía, mientras que otras características hidráulicas, como la vulnerabilidad al embolismo, presentan una variabilidad más limitada (Mencuccini y Bonosi 2001; Poyatos et al. 2007; Martínez-Vilalta et al. 2009). A nivel intra-poblacional, la defoliación seguramente se debe interpretar más como una respuesta inevitable a un balance hídrico negativo que como un ajuste propiamente dicho. La defoliación inducida por sequía se asocia a bajas tasas de crecimiento radial y a niveles muy bajos de reservas de carbohidratos en el tronco. Éstos, a su vez, limitan la capacidad del árbol de recuperar su área foliar y resultan en un aumento en la probabilidad de sucumbir a nuevas sequías (Galiano et al. 2011).

Un aspecto todavía no resuelto es lo que hace que algunos árboles comiencen un proceso de defoliación y decaimiento mientras que sus vecinos de la misma población se mantengan aparentemente sanos. Un trabajo reciente muestra que a nivel hidráulico no existen diferencias importantes entre árboles sanos y defoliados, por lo menos en las ramas (Gómez 2012). En éstas, los niveles de embolismo nativo durante el verano de 2012, extremadamente seco en la zona de estudio de Prades, llegaron al $65 \%$ en promedio, sin que hubiera ninguna diferencia asociada al estado del árbol (Gómez 2012). Sin embargo, sí que encontramos pequeñas diferencias en la vulnerabilidad al embolismo de las raíces, siendo los individuos defoliados ligeramente más vulnerables que los sanos (Gómez 2012). Esta diferencia podría estar asociada a la infección del sistema radicular de los árboles defoliados por hongos patógenos de distintas especies (de momento se han detectado Heterobasidion annosum y Phaeolus schweinitzii; J. Oliva, datos sin publicar) y podría estar en el origen del lento proceso de decaimiento que afecta a las poblaciones estudiadas (cf. Hereş et al. 2012).

Según la hipótesis anterior (Fig. 1), la infección provocaría un consumo directo de carbohidratos de reserva e, indirectamente, una reducción del área fotosintética debida a la mayor constricción hidráulica en las raíces de los árboles afectados. La menor área foliar causaría una reducción en el crecimiento que, a su vez, revertiría en una menor cantidad de tejido conductor y mayores limitaciones al transporte de agua. A la larga, la correspondiente reducción en las reservas de carbono limitaría tanto la capacidad de recuperar área foliar como la capacidad de luchar contra nuevas infecciones o plagas. Estos árboles podrían permanecer en este estado debilitado durante años, hasta llegar a un nivel crítico de afectación que los haría sucumbir ante nuevas sequías. La culminación del proceso comporta la decoloración y pérdida de la totalidad de las hojas y la muerte del árbol, y puede ser relativamente rápido (Fig. 2). La interpretación anterior es coherente con los resultados preliminares de un estudio experimental en condiciones controladas, los cuales sugieren que la reducción en las reservas 


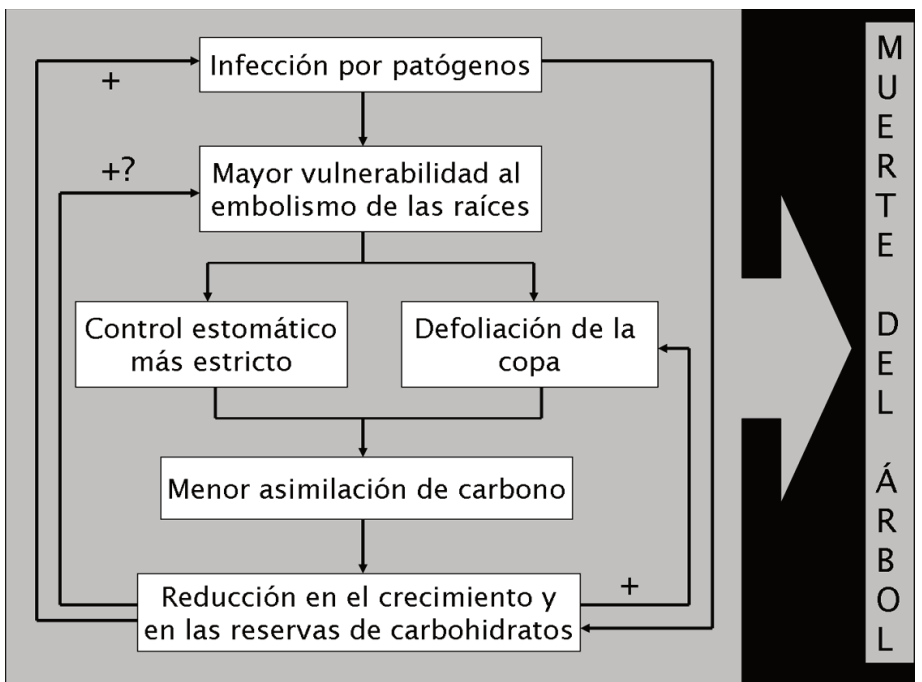

Figura 1. Diagrama que muestra los posibles mecanismos y retroalimentaciones involucradas en el proceso de mortalidad del pino albar, basado en estudios propios realizados en las montañas de Prades (Tarragona).

de carbohidratos es uno más entre los factores que contribuyen al decaimiento en el pino albar y no necesariamente precede a la mortalidad (Garcia et al., resultados no publicados). Hay que tener en cuenta, sin embargo, que la hipótesis esbozada en la Figura 1 es todavía muy preliminar. Se necesitarán estudios adicionales para aclarar algunos aspectos todavía poco claros (e.g., ¿qué es lo que hace a algunos individuos más vulnerables a ser infectados pon hongos patógenos?) y, sobre todo, para ver hasta qué punto el mecanismo propuesto es generalizable a otras poblaciones de pino albar en decaimiento.

\section{Tendencias en el crecimiento}

Desde el punto de vista de los servicios ambientales, tan importante como entender el mecanismo subyacente a la mortalidad inducida por sequía es saber cómo el crecimiento de las masas forestales responde a ésta. Numerosos estudios han mostrado que el crecimiento radial del pino albar es menor en años secos, caracterizados por una reducida pluviosidad en relación a la demanda evaporativa atmosférica (e.g., Bogino et al. 2009; Martínez-Vilalta et al. 2008; 2012, Sánchez-Salguero et al. 2012a). Los estudios an- teriores muestran también que el efecto de la temperatura es variable en función, entre otras cosas, de la aridez de la zona de estudio. Así, en zonas secas las temperaturas elevadas siempre implican una disminución en el crecimiento del pino albar, mientras que en las zonas relativamente húmedas el efecto de la temperatura depende de la disponibilidad de agua: es positivo cuando ésta es elevada y negativo cuando es baja (cf. Martínez-Vilalta et al. 2008).

En cuanto a los patrones temporales, el crecimiento radial del pino albar en Cataluña ha aumentado un $84 \%$ (en términos de área basal) a lo largo del siglo XX, de manera coherente con lo ocurrido para la mayoría de árboles de zonas templadas (Martínez-Vilalta et al. 2008). Este aumento es probablemente debido a una combinación de factores, relacionados con el efecto fertilizador del $\mathrm{CO}_{2}$ y el nitrógeno de origen antrópico y con los cambios en la gestión forestal. Al mismo tiempo, se ha observado un aumento en la variabilidad del crecimiento y en la sensibilidad de éste al clima (Andreu et al. 2007), y una ligera disminución en las tasas de crecimiento a partir de mediados de los años 1970s, coincidiendo con el período más cálido y seco del siglo XX (Martínez-Vilalta et al. 2008). Estos resultados sugieren que el crecimiento del pino albar puede verse reducido sustancialmente si el clima se vuelve todavía más seco. Sin embargo, la magnitud de estas reducciones es difícil de predecir (cf. Sabaté et al. 2002; Keenan et al. 2011 para proyecciones utilizando el modelo forestal Gotilwa), entre otras cosas por la dificultad de modelizar los efectos de fenómenos extremos que previsiblemente serán más frecuentes en el futuro, como sequías, incendios y plagas.

Es importante considerar también que la respuesta del crecimiento al clima depende de la edad de los pinos. Bogino et al. (2009) encontraron que las variables climáticas explicaban una mayor proporción de la variabilidad en el crecimiento en los pinares jóvenes. En otro estudio reciente, mostramos que los árboles de mayor edad presentan una menor resiliencia ante un episodio de sequía extrema (Martínez-Vilalta et al. 2012). En este mismo trabajo se constata también que los individuos de crecimiento más rápido se ven proporcionalmente más afectados por la sequía y, aunque su velocidad de recuperación es también mayor, tardan más tiempo en recuperar las tasas de crecimiento previas al episodio. Los efectos de las características de la parcela fueron menores que los de los atributos individuales y, en general, indicaron que la elevada competencia por los recursos empeora los efectos de la sequía en el crecimiento del pino albar (Martínez-Vilalta et al. 2012).
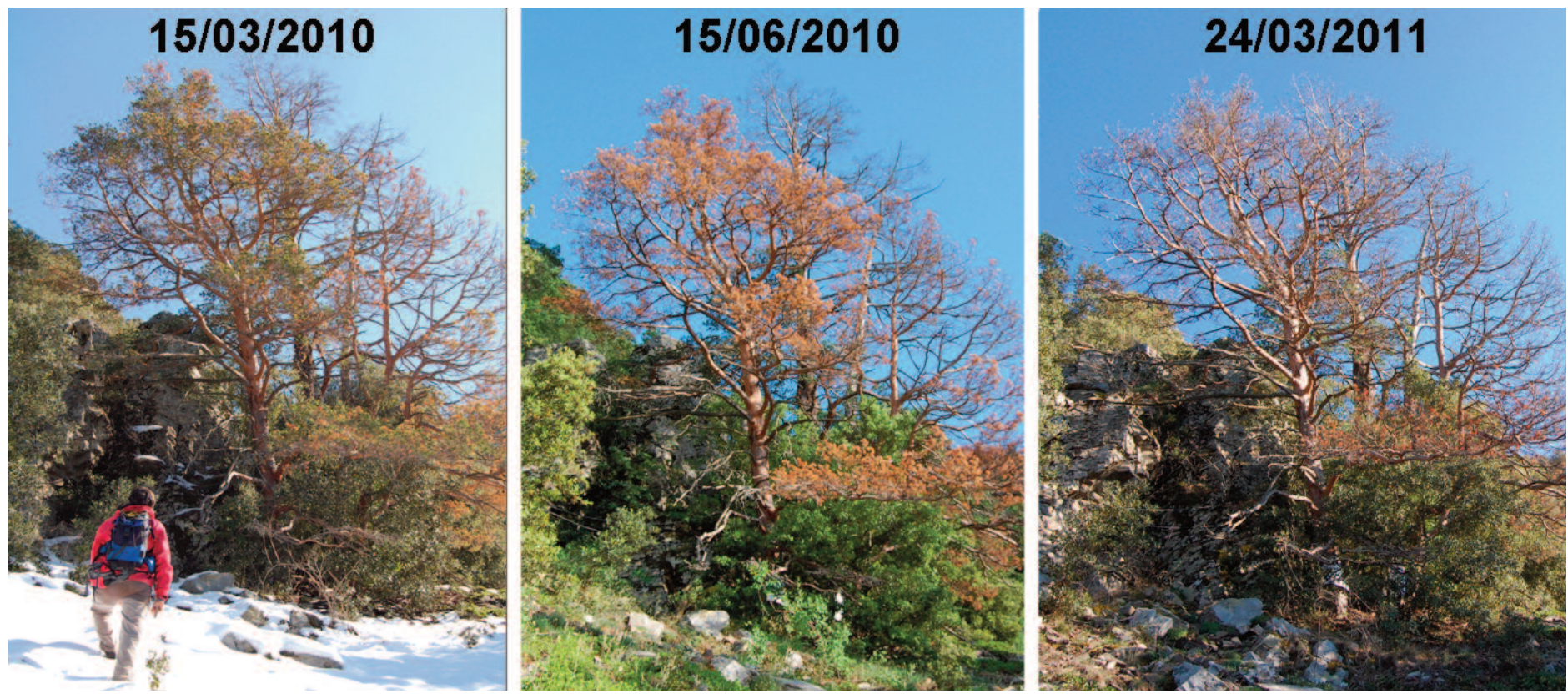

Figura 2. Secuencia de fotografías de un pino albar muriendo en las montañas de Prades (Tarragona). Autores de las fotografías: David Aguadé y Rafael Poyatos. 


\section{Variabilidad espacial en las tasas demográficas}

Desgraciadamente no disponemos de datos que nos permitan evaluar los cambios temporales en las tasas demográficas de mortalidad o reclutamiento tal como hemos visto en el apartado anterior para el crecimiento. Una aproximación habitual en estos casos, aunque evidentemente presenta importantes limitaciones, es utilizar la covariación entre las variables ambientales y las tasas demográficas en el espacio para derivar predicciones temporales (Dunne et al. 2004).

En un estudio reciente, hemos utilizado los datos del Inventario Forestal Nacional (IFN2 e IFN3) para estudiar la distribución espacial de las tasas de crecimiento, mortalidad y reclutamiento de los bosques de pino albar en la Península Ibérica.

Los resultados muestran que la estructura forestal y, en particular, la densidad de pies, es el principal factor que determina la variabilidad espacial de las tasas demográficas del pino albar, lo cual indica un rol preponderante del desarrollo de la masa y de la competencia por los recursos (Vilà-Cabrera et al. 2011). Mortalidad y crecimiento presentaron una covariación negativa en el espacio y parecen responder, en buena medida, a los mismos factores ambientales. En el caso de la mortalidad, la interacción entre el efecto de la estructura forestal y el clima sugiere que la competencia por el agua determina mayores mortalidades en las zonas más secas. Este consideración es especialmente importante si consideramos la gran cantidad de repoblaciones de pino albar con elevadas densidades de pies que existen en nuestro país, muchas de ellas situadas en el límite (o fuera) del nicho climático de la especie (e.g., Sánchez-Salguero et al. 2012b). Finalmente, las tasas de reclutamiento del pino albar son en general bajas, especialmente en las zonas donde se observan mayores mortalidades (Vilà-Cabrera et al. 2011). Un 54 \% de las parcelas del IFN dominadas por el pino albar no presentan reclutamiento de esta especie (Vilà-Cabrera et al. 2011).

No obstante, tomados en conjunto, los resultados disponibles muestran que no estamos (todavía) ante un proceso de decaimiento generalizado del pino albar en la Península lbérica, sino que más allá de los episodios puntuales de mortalidad asociados a condiciones de sequía extremas, los patrones observados se pueden explicar en buena medida por fenómenos de auto-aclareo asociados a la relativa juventud de la mayoría de masas de esta especie. Estos resultados coinciden con las pautas observadas cuando las tasas demográficas (crecimiento, mortalidad, reclutamiento) se expresan como flujos de carbono, los cuales muestran un balance claramente positivo (i.e., una acumulación neta de carbono) (Fig. 3, cf. Vayreda et al. 2012).
No cabe duda tampoco que a escala más local los procesos de decaimiento existen y pueden ser importantes. En el caso del pino albar en la Península Ibérica han sido detectados, entre otras zonas, en las montañas de Prades (con mortalidades del $\sim 20 \%$ de los individuos en pie, Martínez-Vilalta y Piñol 2002), la sierra de los Filabres (Navarro-Cerrillo et al. 2007; Sánchez-Salguero et al. 2012b), o el Pirineo (mortalidades del $\sim 15 \%$ de los individuos en pie, Galiano et al. 2010). En Cataluña, el pino albar es la especie con mayor superficie afectada por decaimiento ( 7600 ha, un $3.6 \%$ de su distribución), según el programa de seguimiento del decaimiento de los bosques en Cataluña (DEBOSCAT, Banqué et al. 2011, datos sin publicar). En general, las tasas de mortalidad elevadas se concentran en zonas con elevada competencia y poca disponibilidad de agua (en relación a la demanda evaporativa atmosférica) y afectan especialmente las zonas donde el calentamiento y/o la reducción en la precipitación observado en las últimas décadas ha sido mayor (Galiano et al. 2010; Vilà-Cabrera et al., en prensa).

A escalas más locales el reclutamiento del pino albar se ve limitado por la disponibilidad de agua en verano (Matías et al. 2012) y es también menor en los rodales que sufren mayor decaimiento, mientras que otras especies, especialmente del género Quercus, se están viendo beneficiadas. Este hecho sugiere que en algunas zonas se podrían estar dando ya las condiciones necesarias para un cambio de vegetación, de bosques de pino albar a bosques de quercíneas (Galiano et al. 2010; 2012). Los impactos funcionales de un cambio de vegetación de este tipo han sido todavía poco estudiados, aunque los trabajos que hemos realizado en las montañas de Prades sugieren que tendrán importantes efectos en los flujos de agua y carbono a nivel del ecosistema (Aguade et al., datos sin publicar; Barba et al., datos sin publicar). Estos últimos parecen mediados por modificaciones sustanciales en la composición y diversidad de la comunidad bacteriana del suelo asociadas a la mortalidad del pino albar (Curiel Yuste et al., en prensa).

\section{Impactos previsibles de un aumento en la sequía y el riesgo de incendios}

Los modelos climáticos proyectan un aumento de las temperaturas de alrededor de $4{ }^{\circ} \mathrm{C}$ y una reducción en las precipitaciones del $20 \%$ en España a lo largo del presente siglo (AEMET 2009), lo cual implicará un aumento considerable en la frecuencia y la intensidad de las sequías. Los cambios actuales en el clima y en la intensidad de las presiones antrópicas son comparables a los ocurridos desde el último máximo glacial y, por tanto, son esperables modificaciones sustanciales en la distribución del pino

\section{Crecimiento (t/ha/año)}

Mortalidad (t/ha/año)

Balance (t/ha/año)

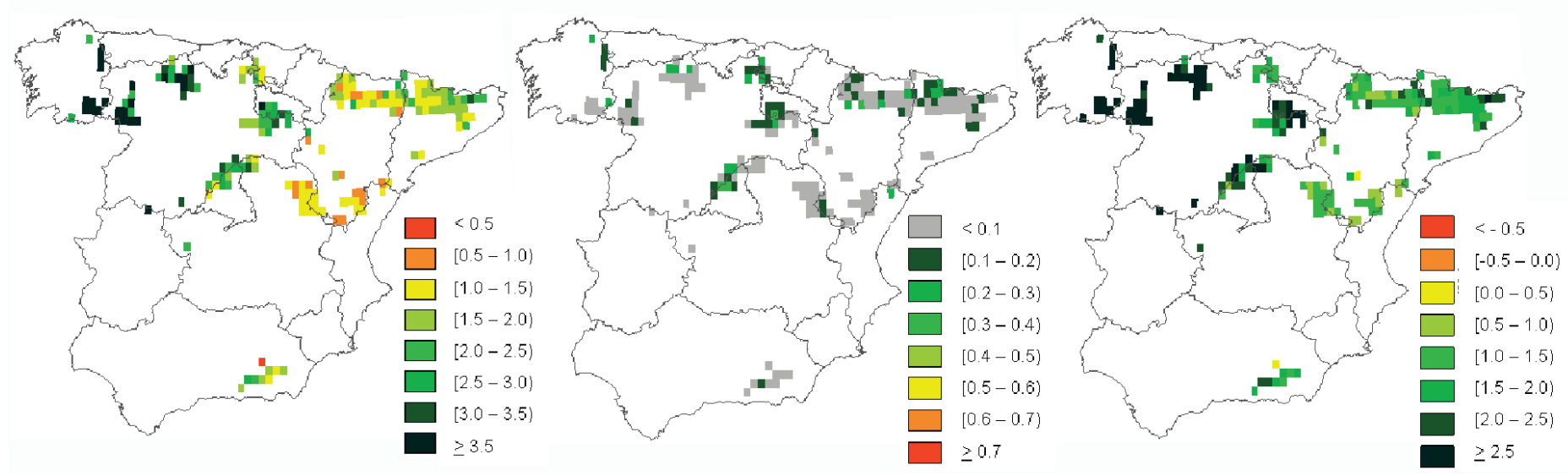

Figura 3. Mapas que muestran el crecimiento, la mortalidad y el balance entre ambos (incluyendo la regeneración) en los bosques de pino albar de la Península Ibérica. En todos los casos las variables se expresan como flujos de carbono, calculadas a partir de los datos del Inventario Forestal Nacional (IFN2 e IFN3). Los valores de cada celda corresponden al promedio de las parcelas del IFN muestreadas en su interior (cf. Vayreda et al. 2012). No se dispone de datos para las Comunidades Autónomas de Asturias, Cantabria y Navarra. 
albar a medio plazo (Matías y Jump 2012). Estos cambios vendrán mediados por los efectos directos de la sequía sobre las poblaciones de pino albar y su efecto acelerador de la dinámica forestal, tal como se ha esbozado en los apartados precedentes, pero también por sus efectos indirectos, a menudo más drásticos a escala local.

Históricamente, los bosques de pino albar españoles han sido poco afectados por incendios forestales. Sin embargo esta situación podría cambiar, puesto que el aumento previsto en las temperaturas resultará en un mayor riesgo climático de incendios. No disponemos de datos para el conjunto de España, pero en Cataluña se quemaron 6700 ha de bosques de pino albar entre 1979 y 2009 (un $3.6 \%$ de la superficie ocupada por la especie). Estos fuegos afectaron principalmente a las zonas más cálidas y secas dentro de la distribución de la especie (Vilà-Cabrera et al. 2012). El 32\% de las poblaciones españolas de pino albar se consideran vulnerables al fuego desde un punto de vista estrictamente climático; porcentaje que aumentaría al $66 \%$ en un escenario de calentamiento climático como el previsto para finales del siglo XXI. La regeneración del pino albar después de los incendios forestales es prácticamente nula, y éstos resultan casi siempre en una sustitución del pino por especies mejor adaptadas al fuego, fundamentalmente robles, encinas y arbustos (Vilà-Cabrera et al. 2012).

Otro aspecto importante son las plagas forestales. Los insectos defoliadores, como la procesionaria del pino (Thaumetopoea pityocampa) o la oruga defoliadora del pino (Lymantria monacha) y los coleópteros barrenadores o perforadores (e.g., géneros Ips, Tomicus) están entre los que causan mayores daños en los pinares ibéricos de pino albar (Gracia y Ordóñez. 2011). Estos últimos son vectores también de hongos patógenos (géneros Leptographium, Ophiostoma), los cuales han sido asociados con el decaimiento del pino albar en poblaciones de los Alpes (Heiniger et al. 2011). El aumento en las temperaturas acelera los ciclos de vida de la mayoría de insectos y se ha relacionado con una mayor incidencia de algunas plagas (e.g., T. pityocampa; Hódar et al. 2003). Por otra parte, se sabe poco sobre los efectos de los hongos patógenos en las poblaciones ibéricas de pino albar en un contexto de cambio climático, más allá de las dos especies de patógenos primarios detectados recientemente en la población de Prades. Finalmente, se han documentado también asociaciones entre los efectos de la sequía y la presencia de muérdago (Viscum album) (e.g., Galiano et al. 2010), así como un importante efecto de la herbivoría por ungulados en poblaciones del sur de España, la cual podría limitar la migración altitudinal de la especie en el límite meridional de su distribución (Herrero et al. 2012).

Los modelos de idoneidad climática o topo-climática sintetizan, en principio, el efecto a largo plazo de la mayoría de factores considerados en los párrafos anteriores, y predicen una reducción drástica ( 90\%) del hábitat adecuado para el pino albar en la Península Ibérica para finales del siglo XXI (Benito-Garzón et al. 2008; Keenan et al. 2011; Ruiz-Labourdette et al. 2012). Estos mismos modelos sugieren que al menos un $25 \%$ de las zonas actualmente ocupadas por el pino albar podrían dejar de ser habitables para la especie en el periodo 2050-2080, manteniéndose la zona de Pirineos como único gran núcleo de distribución (Keenan et al. 2011). De todos modos, el valor predictivo de estos modelos es muy limitado a escala local, puesto que, entre otras cosas, no consideran la variabilidad genética y fenotípica que existe entre poblaciones.

\section{Síntesis e implicaciones para la gestión}

Los bosques de la Península Ibérica en general, y los pinares de pino albar en particular, se encuentran en un momento muy dinámico, determinado tanto por el cambio en el clima como por el abandono de la gestión forestal en muchas zonas. Estos elementos de cambio se han de tener en cuenta necesariamente a la hora de plantear estrategias de gestión. En este contexto, es esencial pre- decir qué poblaciones y qué individuos son más vulnerables a la sequía. Esta vulnerabilidad responde a procesos distintos dependiendo de la escala de estudio. Así, parece claro que dentro del área de distribución de una especie las poblaciones que ocupan lugares más secos son generalmente más vulnerables. Por el contrario, a escala más local dominan aspectos relacionados con la estructura forestal y la predicción es en general más compleja. A esta escala es imprescindible considerar aspectos como la adaptación local de cada población, así como la variabilidad fenotípica entre poblaciones y su plasticidad ante cambios ambientales (Nicotra et al. 2010; cf. Laforest et al., datos sin publicar, para el caso del pino albar). A escalas todavía más detalladas es imprescindible integrar también los aspectos hidrológicos, relacionados tanto con la disponibilidad de agua a nivel climático como con la disponibilidad edáfica, determinada por la topografía y, sobre todo, por la profundidad y textura del suelo.

Diversos estudios muestran la importancia de la estructura forestal en los efectos de la sequía sobre las poblaciones de pino albar. No cabe duda, por tanto, que actuaciones silvícolas orientadas a disminuir la densidad de pies (y por tanto la competencia) pueden ser una herramienta útil para disminuir la vulnerabilidad de determinadas masas, tanto a la sequía como a algunas plagas. Es especialmente importante adaptar las prácticas silvícolas para reducir al máximo el riesgo de infección por hongos patógenos (e.g., H. annosum) y el ataque de plagas (Hódar et al. 2012). En los bosques explotados, las rotaciones largas resultan en un mayor almacenamiento de carbono, lo cual se podría traducir en un beneficio económico nada desdeñable en muchas zonas. En relación a los incendios, parece claro que las masas de pino albar se deben considerar como susceptibles al fuego en las condiciones climáticas actuales y futuras y, por tanto, se les deberían aplicar las estrategias de prevención que hasta ahora se han limitado a bosques de zonas más secas.

Hay que considerar, de todos modos, que si las proyecciones climáticas se cumplen una proporción importante de las poblaciones actuales de pino albar de la Península Ibérica estarían condenadas a desaparecer a medio plazo. Incluso bajo escenarios de cambio climático moderado, la dinámica natural de sucesión en muchos bosques de pino albar debería conducir a su reemplazamiento por planifolios (particularmente especies del género Quercus), a no ser que se vuelvan a aplicar prácticas de aclareo selectivo que actualmente no son viables económicamente. En este contexto, parece razonable concentrar los esfuerzos de gestión en las zonas que se encuentren claramente dentro del nicho climático de la especie y en función también del tipo de vegetación que potencialmente reemplazaría al pino albar. En zonas concretas, existe la posibilidad de favorecer la migración altitudinal (o latitudinal) de la especie hacia climas menos áridos mediante plantaciones. En ese caso es muy importante considerar la procedencia del material que se utilice en la plantación, asegurando, hasta donde sea posible, que corresponde a una población suficientemente adaptada a la sequía (Alía et al. 2001; García-López et al. 2010).

\section{Agradecimientos}

Muchos de los estudios en los que se basa este trabajo hubieran sido imposibles sin la colaboración del personal del Paratge Natural d'Interès Nacional de Poblet y del Parc Natural de l'Alt Pirineu. Queremos agradecer también la colaboración de Maurizio Mencuccini y Jonàs Oliva en los trabajos llevados a cabo en las montañas de Prades, y de todas las personas implicadas en la elaboración de las distintas ediciones del Inventario Forestal Nacional. Los estudios realizados han sido posibles gracias a los proyectos CGL2006-01293, CGL2007-60120, CSD2008-0004, CGL200908101 y CGL2010-16373, financiados por el del Ministerio de Ciencia e Innovación, y SGR2009-0247, financiado por la Generalitat de Catalunya. 


\section{Referencias}

AEMET 2009. Generación de escenarios regionalizados de cambio climático para España. Agencia Estatal de Meteorología, Ministerio de Medio Ambiente y Medio Rural y Marino, Madrid, España.

Alía, R., Moro-Serrano, J., Notivol, E. 2001. Genetic variability of Scots pine (Pinus sylvestris) provenances in Spain: growth traits and survival. Silva Fennica 35:27-38.

Allen, C.D., Macalady, A.K., Chenchouni, H., Bachelet, D., McDowell, N., Vennetier, M., Kitzberger, T., Rigling, A., Breshears, D.D., Hogg, E.H., Gonzalez, P., Fensham, R., Zhang, Z., Castro, J., Demidova, N., Lim, J.-H., Allard, G., Running, S.W., Semerci, A., Cobb, N. 2010. A global overview of drought and heat-induced tree mortality reveals emerging climate change risks for forests. Forest Ecology and Management 259:660-684.

Anderegg, W.R.L., Kane, J., Anderegg, L.D.L. 2012. Consequences of widespread tree mortality triggered by drought and temperature stress. Nature Climate Change, doi:10.1038/nclimate1635.

Andreu, L., Gutiérrez, E., Macias, M., Ribas, M., Bosch, O., Camarero, J.J. 2007. Climate increases regional tree-growth variability in Iberian pine forests. Global Change Biology 13:804-815.

Benito-Garzón, M., Sánchez, R., Sainz, H. 2008. The evolution of the Pinus sylvestris $\mathrm{L}$. area in the Iberian Peninsula from the last glacial maximum to 2100 under climate change. The Holocene 18:705-714.

Bogino, S., Fernández Nieto, M.J., Bravo, F. 2009. Climate Effect on Radial Growth of Pinus sylvestris at Its Southern and Western Distribution Limits. Silva Fennica 43:609-623.

Bonan, G.B. 2008. Forests and Climate Change: Forcings, Feedbacks, and the Climate Benefits of Forests. Science 320:1444-1449.

Cheddadi, R., Vendramin, G.G., Litt, T., François, L., Kageyama, M., Lorentz, S., Laurent, J., de Beaulieu, J., Sadori, L., Jost, A., Lunt, D. 2006 Imprints of glacial refugia in the modern genetic diversity of Pinus sylvestris. Global Ecology and Biogeography 15:271-282.

Curiel Yuste, J., Barba, J., Fernandez-Gonzalez, J., Fernandez-Lopez, M., Mattana, S., Martinez-Vilalta, J., Nolis, P., Lloret, F. 2012, en prensa. Changes in soil bacterial community triggered by drought-induced gap succession preceded changes in soil $\mathrm{C}$ stocks and quality. Ecology and Evolution 00:000-000.

Dunne, J.A., Saleska, S.R., Fischer, M.L., Harte, J. 2004. Integrating experimental and gradient methods in ecological climate change research. Ecology 85:904-916.

Galiano, L., Martínez-Vilalta, J., Lloret, F. 2010. Drought-Induced Multifactor Decline of Scots Pine in the Pyrenees and Potential Vegetation Change by the Expansion of Co-occurring Oak Species. Ecosystems 13:978-991.

Galiano, L., Martínez-Vilalta, J., Lloret, F. 2011. Carbon reserves and canopy defoliation determine the recovery of Scots pine $4 \mathrm{yr}$ after a drought episode. New Phytologist 190:750-759.

Galiano, L., Martínez-Vilalta, J., Eugenio, M., Granzow de la Cerda, I. Lloret, F. 2012, en prensa. Seedling emergence and growth of Quercus spp. following severe drought effects on a Pinus sylvestris canopy. Journal of Vegetable Science 00:000-000.

García López, J.M., Allué Camacho, C. 2010. Effects of climate change on the distribution of Pinus sylvestris L. stands in Spain. A phytoclimatic approach to defining management alternatives. Forest Systems 19:329-339.

Gómez, M. 2012. Hydraulic properties are unrelated to differences in drought-induced defoliation in Scots pine (Pinus sylvestris L.). Trabajo final de master, UAB.

Gracia, M., Ordóñez, J.L. (eds.) 2011. Les pinedes de pi riog. Manuals de gestió d'hàbitats. Diputació de Barcelona, Barcelona, España.

Hampe, A., Petit, R.J. 2005. Conserving biodiversity under climate change: the rear edge matters. Ecology Letters 5:461-467.

Heiniger, U., Theile, F., Rigling, A., Rigling, D. 2011. Blue-stain infections in roots, stems and branches of declining Pinus sylvestris trees in a dry inner alpine valley in Switzerland. Forest Pathology 41:501-509.

Hereş, A.-M., Martínez-Vilalta, J., Claramunt López, B. 2012. Growth patterns in relation to drought-induced mortality at two Scots pine (Pinus sylvestris L.) sites in NE Iberian Peninsula. Trees 26:621-630.

Herrero, A., Zamora, R., Castro, J., Hódar, J.A. 2012. Limits of pine forest distribution at the treeline: herbivory matters. Plant Ecol. Doi. 10.1007/s11258-011-9993-0.

Hódar, J.A., Castro, J., Zamora, R. 2003. Pine processionary caterpillar Thaumetopoea pityocampa as a new threat for relict Mediterranean Scots pine forests under climatic warming. Biological Conservation 110:123-129

Hódar, J.A., Zamora, R., Cayuela, L. 2012. Cambio climático y plagas: algo más que el clima. Ecosistemas 21(3):73-78.
Irvine, J., Perks, M., Magnani, F., Grace, J. 1998. The response of Pinus sylvestris to drought: stomatal control of transpiration and hydraulic conductance. Tree Physiology 18:393-402.

Keenan, T., Serra, J.M., Lloret, F., Ninyerola, M., Sabaté, S. 2011. Predicting the future of forests in the Mediterranean under climate change, with niche- and process-based models: $\mathrm{CO}_{2}$ matters! Global Change Biology 17:565-579.

Lloret, F. 2012. Vulnerabilidad y resiliencia de los ecosistemas terrestres frente a episodios extremos de sequía. Ecosistemas 21(3):85-90.

Lloret, F., Escudero, A., Iriondo, J.M., Martínez-Vilalta, J., Valladares, F. 2012. Extreme climatic events and vegetation: the role of stabilizing processes. Global Change Biology 18:797-805.

Martínez-Vilalta, J., Piñol, J. 2002. Drought-induced mortality and hydraulic architecture in pine populations of the NE Iberian Peninsula. Forest Ecology and Management 161:247-256.

Martínez-Vilalta, J., López, B.C., Adell, N., Badiella, L., Ninyerola, M. 2008. Twentieth century increase of Scots pine radial growth in NE Spain shows strong climate interactions. Global Change Biology 14:28682881.

Martínez-Vilalta, J., Cochard, H., Mencuccini, M., Sterck, F., Herrero, A. Korhonen, J.F.J., Llorens, P., Nikinmaa, E., Nolè, A., Poyatos, R., Ripullone, F., Sass-Klaassen, U., Zweifel, R. 2009. Hydraulic adjustment of Scots pine across Europe. New Phytologist 184:353-364.

Martínez-Vilalta, J., López, B.C., Loepfe, L., Lloret, F. 2012. Stand- and treelevel determinants of the drought response of Scots pine radial growth. Oecologia 168:877-888.

Mason, W.L., Alía, R. 2000. Current and future status of Scots pine (Pinus sylvestris L.) forests in Europe. Investigación Agraria: Sistemos y Recursos Forestales. Fuera de Serie 1:317-336.

Matías, L., Jump, A.S. 2012. Interactions between growth, demography and biotic interactions in determining species range limits in a warming world: The case of Pinus sylvestris. Forest Ecology and Management 282:10-22.

Matías, L, Zamora, R., Castro, J. 2012. Sporadic rainy events are more critical than increasing of drought intensity for woody species recruitment in a Mediterranean Community. Oecologia 169:833-844.

McDowell, N., Pockman, W.T., Allen, C.D., Breshears, D.D., Cobb, N., Kolb, T., Plaut, J., Sperry, J., West, A., Williams, D.G. 2008. Mechanisms of plant survival and mortality during drought: why do some plants survive while others succumb to drought? New Phytologist 178:719-739.

McDowell, N.G. 2011. Mechanisms Linking Drought, Hydraulics, Carbon Metabolism, and Vegetation Mortality. Plant Physiology 155:1051-1059.

McDowell, N.G., Beerling, D.J., Breshears, D.D., Fisher, R.A., Raffa, K.F., Stitt, M. 2011. The interdependence of mechanisms underlying climate-driven vegetation mortality. Trends in Ecology and Evolution 26:523-532.

Mencuccini, M., Bonosi, L. 2001. Leaf/sapwood area ratios in Scots pine show acclimation across Europe. Canadian Journal of Forest Research 31:442-456.

Navarro-Cerrillo, R.M., Varo, M.A., Lanjeri, S., Hernández Clemente, R. 2007. Cartografía de defoliación en los pinares de pino silvestre (Pinus sylvestris L.) y pino salgareño (Pinus nigra Arnold.) en la Sierra de los Filabres. Ecosistemas 2007(3):163-171.

Nicotra, A.B., Atkin, O.K., Bonser, S.P., Davidson, A.M., Finnegan, E.J., Mathesius, U., Poot, P., Purugganan, M.D., Richards, C.L., Valladares, F., van Kleunen, M. 2010. Plant phenotypic plasticity in a changing climate. Trends in Plant Science 15:684-692.

Pan, Y., Birdsey, R.A., Fang, J., Houghton, R., Kauppi, P.E., Kurz, W.A., Phillips, O.L., Shvidenko, A., Lewis, S.L., Canadell, J.G., Ciais, P., Jackson, R.B., Pacala, S.W., McGuire, A.D., Piao, S., Rautiainen, A., Sitch, S., Hayes, D. 2011. A large and persistent carbon sink in the World's forests. Science 333:988-993.

Poyatos, R., Martínez-Vilalta, J., Čermák, J., Ceulemans, R., Granier, A., Irvine, J., Köstner, B., Lagergren, F., Meiresonne, L., Nadezhdina, N. Zimmermann, R., Llorens, P., Mencuccini, M. 2007. Plasticity in hydraulic architecture of Scots pine across Eurasia. Oecologia 153:245-259.

Ruiz-Labourdette, D., Nogues-Bravo, D., Sainz Ollero, H, Schmitz, M.F., Pineda, F.D. 2012. Forest composition in Mediterranean mountains is projected to shift along the entire elevational gradient under climate change. Journal of Biogeography 39:162-176.

Sabaté, S., Gracia, C.A., Sánchez, A. 2002. Likely effects of climate change on growth of Quercus ilex, Pinus halepensis, Pinus pinaster, Pinus sylvestris and Fagus sylvatica forests in the Mediterranean region. Forest Ecology and Management 162:23-37.

Sánchez-Salguero, R., Navarro-Cerrillo, R.M., Camarero, J.J., FernándezCancio, A., Swetnam, T.W., Zavala, M.A. 2012a. Vulnerabilidad frente a la sequía de repoblaciones de dos especies de pinos en su límite meridional en Europa. Ecosistemas 21(3):31-40. 
Sanchez-Salguero, R., Navarro-Cerrillo, R.M., Swetnam, T.W., Zavala, M.A $2012 \mathrm{~b}$. Is drought the main decline factor at the rear edge of Europe? The case of southern Iberian pine plantations. Forest Ecology and Management 271:158-169.

Sala, A., Piper, F., Hoch, G. 2010. Physiological mechanisms of drought-induced tree mortality are far from being resolved. New Phytologist 186:274-281.

Vilà-Cabrera, A., Martínez-Vilalta, J., Vayreda, J., Retana, J. 2011. Structural and climatic determinants of demographic rates of Scots pine forests across the Iberian Peninsula. Ecological Applications 21:11621172.

Vilà-Cabrera, A., Martínez-Vilalta, J., Galiano, L., Retana, J. 2012, en prensa. Patterns of forest decline and regeneration across Scots pine populations. Ecosystems 00:000-000.
Vila-Cabrera, A., Rodrigo, A., Martinez-Vilalta, J., Retana, J. 2012. Lack of regeneration and climatic vulnerability to fire of Scots pine may induce vegetation shifts at the southern edge of its distribution. Journal of Biogeography 39:488-496.

Vayreda, J., Martinez-Vilalta, J., Gracia, M., Retana, J. 2012. Forest structure and management interact with recent changes in climate to determine the current forest carbon stock in Peninsular Spain. Global Change Biology 18:1028-1041.

Willis, K.J., Bennett, K.D., Birks, H.J.B. 1998. The late Quaternary dynamics of pines in Europe. En: Richardson, D.M. (ed.), Ecology and Biogeography of Pinus, pp. 107-121, Cambridge University Press, Cambridge, UK.

Zweifel, R., Steppe, K., Sterck, F.J. 2007. Stomatal regulation by microclimate and tree water relations: interpreting ecophysiological field data with a hydraulic plant model. Journal of Experimental Botany 58:2113-2131. 